\title{
Reliability Analysis of the Chatter Stability during Milling Using a Neural Network
}

\author{
Sen Hu, Xianzhen Huang, Yimin Zhang, and Chunmei Lv \\ School of Mechanical Engineering and Automation, Northeastern University, Shenyang 110819, China \\ Correspondence should be addressed to Xianzhen Huang; xzhhuang83@gmail.com
}

Received 28 July 2016; Accepted 19 September 2016

Academic Editor: Christopher J. Damaren

Copyright ( 92016 Sen Hu et al. This is an open access article distributed under the Creative Commons Attribution License, which permits unrestricted use, distribution, and reproduction in any medium, provided the original work is properly cited.

\begin{abstract}
The parameters of a system have the randomness generally in the process of milling, which influences the stability of the milling. This paper uses the neural network to get a comprehensive analysis of the influences of random factors in milling and proposes a method for reliability analysis of the regenerative chatter stability in milling. Dynamic model of milling regenerative chatter is established, and stability lobe diagram is obtained by the full-discretization method (FDM). The neural network is applied to approximate the functional relationship of the limit axial cutting depth; then the reliability is computed with the Monte Carlo simulation method (MCSM) and the moment method (MM), respectively. Finally, the results of an example are used to demonstrate the efficiency and accuracy of the proposed method.
\end{abstract}

\section{Introduction}

High-speed milling processes are widely employed in the aerospace, automobile, ship building, and energy industry, among other fields, because they offer a high precision, high surface quality, and high material removal rate. However, in recent years, with the rapid development of modern industry and the ever increasing pressure of market competition, mechanical products have shown a tendency to become more and more special. Yet, complex shapes, thin-walled structures, the use of materials that are difficult to process, and high accuracy requirements all increase the probability of the occurrence of vibrations during product processing. This will ultimately affect the processing quality and reduce production efficiency. In more serious cases, production accidents might occur and threaten the safety of the personnel and thus the security of the enterprise. Accordingly, research on the origin of vibrations during milling processes has become extremely important.

Chatter is a kind of self-excited vibration, which is often caused by a regenerative feedback occurring in the system. Four different types of chatter vibrations can be distinguished according to the different feedback modes: regenerative chatter [1], mode-coupling chatter [2], frictional chatter [3], and thermal chatter [4]. In milling processes, regenerative chatter is the main cause of instabilities during the machining process, effectively reducing the quality of the produced parts [5].

Various methods have been reported in the literature to predict the chatter stability. In general, they can be classified into two categories: numerical methods [6-8] and analytical (or semianalytical) methods [9-22]. Sridhar et al. [6] proposed a numerical algorithm to analyze the milling stability. Smith and Tlusty [7] suggested using peak-to-peak (PTP) diagrams for analyzing the milling stability in the time-domain. Campomanes and Altintas [8] later improved the time-domain simulation model and proposed using the ratio of the dynamic uncut chip thickness to the static uncut chip thickness as an instability criterion. Based on the theory of periodic differential equations, Minis and Yanushevsky [9] employed the Nyquist criterion to calculate the stable boundary of the milling process. Altintaş and Budak [10] introduced the zeroth-order approximation (ZOA) method. This method has a high computational efficiency, but it cannot be used to predict the flip bifurcation in low radial immersion milling. Merdol and Altintas [11] then applied the 


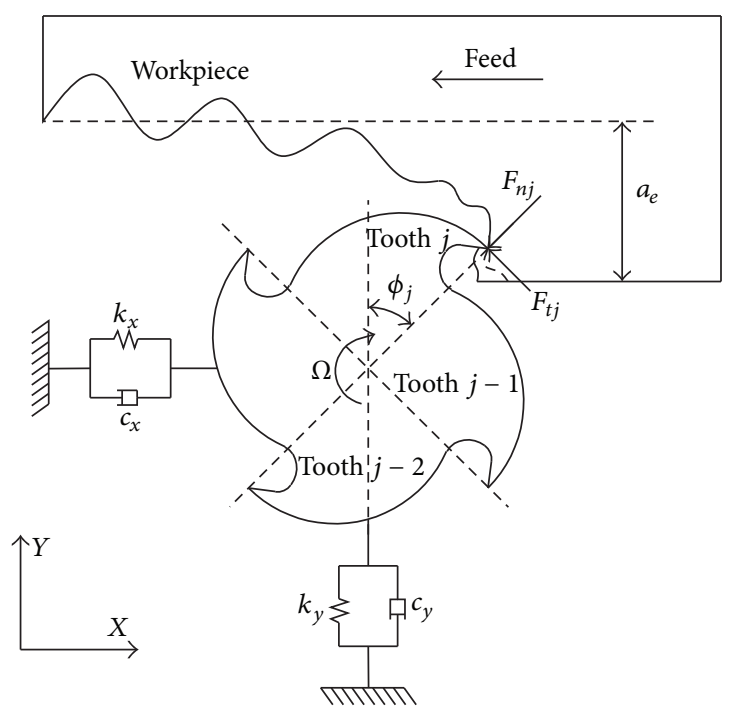

FIgURE 1: Dynamic model of the milling process.

multifrequency method to solve this problem. Bayly et al. [12] proposed the temporal finite element analysis (TFEA) method. The TFEA method can be applied to simultaneously predict the milling stability and the machining error. Insperger and Stépán [13] presented the semidiscretization method (SDM) for milling stability analysis. To improve the calculation accuracy, they then proposed the first-order SDM [14]. Ding et al. [15] established the full-discretization method (FDM) for milling stability analysis, which is based on the immediate integration method. The FDM can also simultaneously predict the milling stability and the machining error. Another kind of FDM is presented by Li et al. [16]. All the time-dependent terms are discretized in this method, and the transition matrix is obtained by numerical iteration method. Based on the TFEA method, Ding et al. [17] used integral equation technique to calculate the response of dynamic milling system and proposed the numerical integration method. Subsequently, they proposed a timedomain semianalytical method for stability analysis of milling in the framework of the differential quadrature method [18]. Besides, there are some other methods, such as [19-22]. Albertelli et al. [23] built a model for the stability analysis of a heavy milling machine and optimized both the planning process and the tool selection process.

The above-mentioned methods can be considered as a foundation for the analysis and the control of milling chatter. However, these methods are based on fixed cutting parameters. But in the actual machining process, the cutting parameters may vary and therefore must be treated as uncertain and random variables [24]. For example, variations in the working environment (such as voltage fluctuations) can make the feed of the workpiece random. Consequentially, the resulting measurement errors, machining errors, and other errors will lead to variations of the geometric dimensions of the tool. Kurdi et al. [25] studied the uncertainty in stability and surface location error in high-speed milling. Duncan et al. [26] described a procedure for adding uncertainty bounds to the analytical milling stability limits by the Monte Carlo simulation method (MCSM). Graham et al. [27] utilized the edge theorem and the zero exclusion condition to develop a robust chatter stability model based on the analytical chatter stability milling model. The reliability of milling chatter system was calculated using the first-order secondmoment method (FOSMM) and compared to the Monte Carlo simulation method (MCSM) [28]. Lin [29] described the reliability of the cutting tools in the high-speed machining by normal distribution model.

In this study, we analyzed the milling stability using the FDM, and an artificial neural network was employed to analyze the effect of random factors on the milling stability. Furthermore, a method for calculating the reliability of the milling stability was developed and is presented in this paper.

\section{Milling Chatter Stability Analysis}

The dynamic model typically used for describing milling processes is shown in Figure 1. In this figure, $\phi_{j}$ is the angular position of $j$ th tooth, $\Omega$ is the spindle speed ( $\mathrm{r} / \mathrm{min}), a_{e}$ is the radial cutting depth, and $F_{t j}$ and $F_{n j}$ are the tangential and normal components of the cutting force for $j$ th tooth, respectively.

The governing equation of the dynamic milling system shown in Figure 1 can be expressed as [30]

$$
\mathbf{M} \ddot{\mathbf{q}}(t)+\mathbf{C} \dot{\mathbf{q}}(t)+\mathbf{K q}(t)=\mathbf{K}_{c}(t)[\mathbf{q}(t)-\mathbf{q}(t-T)],
$$

where $\mathbf{M}, \mathbf{C}$, and $\mathbf{K}$ represent the modal mass matrix, damping matrix, and stiffness matrix, respectively. $\mathbf{q}(t)$ is the modal vector of the cutter. $T$ is the time delay: that is, $T=60(N \Omega)^{-1}$, where $N$ is the number of cutting teeth. $\mathbf{K}_{c}(t)$ denotes the cutting coefficient matrix which varies 
periodically with time: that is, $\mathbf{K}_{c}(t)=\mathbf{K}_{c}(t+T)$, which can be written as

$$
\mathbf{K}_{c}(t)=-a_{p}\left[\begin{array}{ll}
h_{x x}(t) & h_{x y}(t) \\
h_{y x}(t) & h_{y y}(t)
\end{array}\right],
$$

where $a_{p}$ is the axial cutting depth and $h_{x x}(t), h_{x y}(t), h_{y x}(t)$, and $h_{y y}(t)$ are the cutting coefficients given by

$$
\begin{aligned}
& h_{x x}(t)=\sum_{j=1}^{N} w\left(\phi_{j}(t)\right) s \phi_{j}\left[K_{t} c \phi_{j}+K_{n} s \phi_{j}\right], \\
& h_{x y}(t)=\sum_{j=1}^{N} w\left(\phi_{j}(t)\right) c \phi_{j}\left[K_{t} c \phi_{j}+K_{n} s \phi_{j}\right], \\
& h_{y x}(t)=\sum_{j=1}^{N} w\left(\phi_{j}(t)\right) s \phi_{j}\left[-K_{t} s \phi_{j}+K_{n} c \phi_{j}\right], \\
& h_{y y}(t)=\sum_{j=1}^{N} w\left(\phi_{j}(t)\right) c \phi_{j}\left[-K_{t} s \phi_{j}+K_{n} c \phi_{j}\right],
\end{aligned}
$$

where $s \phi_{j}=\sin \left(\phi_{j}(t)\right), c \phi_{j}=\cos \left(\phi_{j}(t)\right) . K_{n}$ and $K_{t}$ are the linearized normal and tangential cutting coefficients, respectively. $\phi_{j}(t)$ is defined by

$$
\phi_{j}(t)=\left(\frac{2 \pi \Omega}{60}\right) t+\frac{2 \pi(j-1)}{N}
$$

and $w\left(\phi_{j}\right)$ is the window function given by

$$
w\left(\phi_{j}\right)= \begin{cases}1, & \text { if } \phi_{s} \leq \phi_{j} \leq \phi_{e} \\ 0, & \text { otherwise, }\end{cases}
$$

where $\phi_{s}$ and $\phi_{e}$ are the start and exit angles of $j$ th tooth, respectively. For climb (down) milling, $\phi_{s}=\arccos \left(2 r_{c}-1\right)$ and $\phi_{e}=\pi$; for conventional (up) milling, $\phi_{s}=0$ and $\phi_{e}=\arccos \left(1-2 r_{c}\right)$, where $r_{c}$ is the radial immersion ratio which is defined by $r_{c}=a_{e} / D$, where $D$ is the cutter diameter.

Assuming that $\mathbf{p}(t)=\mathbf{M} \dot{\mathbf{q}}(t)+\mathbf{C q}(t) / 2$ and $\mathbf{x}(t)=$ $\left[\begin{array}{ll}\mathbf{q}(t) & \mathbf{p}(t)\end{array}\right]^{T}$, (1) can be transformed into the state-space form

$$
\dot{\mathbf{x}}(t)=\mathbf{A x}(t)+\mathbf{B}(t)(\mathbf{x}(t)-\mathbf{x}(t-T)),
$$

where

$$
\begin{aligned}
\mathbf{A} & =\left[\begin{array}{cc}
-\frac{\mathbf{M}^{-1} \mathbf{C}}{2} & \mathbf{M}^{-1} \\
\frac{\mathbf{C M}^{-1} \mathbf{C}}{4}-\mathbf{K} & -\frac{\mathbf{C M}^{-1}}{2}
\end{array}\right], \\
\mathbf{B}(t) & =\left[\begin{array}{cc}
0 & 0 \\
\mathbf{K}_{c}(t) & 0
\end{array}\right] .
\end{aligned}
$$

In the FDM [15], the first step is to discretize the time by dividing the period $T$ into $m$ equal parts; that is, $T=m \Delta t$ (with $m \in Z$ ). For a given time, (6) can be reformulated as

$$
\dot{\mathbf{y}}(t)=\mathbf{A y}(t)+\widetilde{\mathbf{B}}(t)(\widetilde{\mathbf{y}}(t)-\widetilde{\mathbf{y}}(t-T)),
$$

$$
t \in\left[t_{j}, t_{j+1}\right]
$$

where $t_{j}=j \Delta t$ (with $j \in Z$ ). The periodic-coefficient item $\widetilde{\mathbf{B}}(t)$, the state item $\widetilde{\mathbf{y}}(t)$, and the time delay item $\widetilde{\mathbf{y}}(t-T)$ can be determined through linear approximation:

$$
\begin{gathered}
\widetilde{\mathbf{B}}(t)=\mathbf{B}_{j+1}+\frac{\mathbf{B}_{j+1}-\mathbf{B}_{j}}{\Delta t}\left(t-t_{j+1}\right), \\
\widetilde{\mathbf{y}}(t)=\mathbf{y}_{j+1}+\frac{\mathbf{y}_{j+1}-\mathbf{y}_{j}}{\Delta t}\left(t-t_{j+1}\right), \\
\widetilde{\mathbf{y}}(t-T)=\mathbf{y}_{j-m+1}+\frac{\mathbf{y}_{j-m+1}-\mathbf{y}_{j-m}}{\Delta t}\left(t-t_{j+1}\right)
\end{gathered}
$$

with $\mathbf{B}_{j}=\mathbf{B}\left(t_{j}\right), \mathbf{y}_{j}=\mathbf{y}\left(t_{j}\right)$. Treating (8) as an ordinary differential equation (ODE) and solving over the discretization period $\left[t_{j}, t_{j+1}\right]$ yield

$$
\begin{aligned}
\mathbf{y}_{j+1}= & \left(\boldsymbol{\Phi}_{0}+\mathbf{F}_{j}\right) \mathbf{y}_{j}+\mathbf{F}_{j+1} \mathbf{y}_{j+1}-\mathbf{F}_{j+1} \mathbf{y}_{j-m+1} \\
& -\mathbf{F}_{j} \mathbf{y}_{j-m},
\end{aligned}
$$

where

$$
\begin{aligned}
& \mathbf{F}_{j}=\frac{\Phi_{3}}{\Delta t^{2}} \mathbf{B}_{j}+\left(\frac{\Phi_{2}}{\Delta t}-\frac{\Phi_{3}}{\Delta t^{2}}\right) \mathbf{B}_{j+1}, \\
& \mathbf{F}_{j+1}=\left(\frac{\Phi_{2}}{\Delta t}-\frac{\Phi_{3}}{\Delta t^{2}}\right) \mathbf{B}_{j}+\left(\Phi_{1}-2 \frac{\Phi_{2}}{\Delta t}+\frac{\Phi_{3}}{\Delta t^{2}}\right) \mathbf{B}_{j+1} \\
& \Phi_{0}=e^{\mathbf{A} \Delta t}, \\
& \Phi_{1}=\mathbf{A}^{-1}\left(\Phi_{0}-\mathbf{I}\right), \\
& \Phi_{2}=\mathbf{A}^{-1}\left(\Delta t \Phi_{0}-\Phi_{1}\right), \\
& \boldsymbol{\Phi}_{3}=\mathbf{A}^{-1}\left(\Delta t^{2} \boldsymbol{\Phi}_{0}-2 \Phi_{2}\right) \text {. }
\end{aligned}
$$

In (13), $\mathbf{I}$ is the identity matrix.

According to (10), a discrete map can be defined as

$$
\mathbf{z}_{j+1}=\mathbf{D}_{j} \mathbf{z}_{j}
$$

where 


$$
\begin{aligned}
\mathbf{z}_{j} & =\operatorname{col}\left(\mathbf{y}_{j}, \mathbf{y}_{j-1}, \ldots, \mathbf{y}_{j+1-m}, \mathbf{y}_{j-m}\right) \\
\mathbf{D}_{j} & =\left[\begin{array}{cccccc}
{\left[\mathbf{I}-\mathbf{F}_{j+1}\right]^{-1}\left(\mathbf{\Phi}_{0}+\mathbf{F}_{j}\right)} & \mathbf{0} & \cdots & \mathbf{0} & -\left[\mathbf{I}-\mathbf{F}_{j+1}\right]^{-1} \mathbf{F}_{j+1}-\left[\mathbf{I}-\mathbf{F}_{j+1}\right]^{-1} \mathbf{F}_{j} \\
\mathbf{I} & \mathbf{0} & \cdots & \mathbf{0} & \mathbf{0} & \mathbf{0} \\
\mathbf{0} & \mathbf{I} & \cdots & \mathbf{0} & \mathbf{0} & \mathbf{0} \\
\vdots & \vdots & \ddots & \vdots & \vdots & \vdots \\
\mathbf{0} & \mathbf{0} & \cdots & \mathbf{I} & \mathbf{0} & \mathbf{0} \\
\mathbf{0} & \mathbf{0} & \cdots & \mathbf{0} & \mathbf{I} & \mathbf{0}
\end{array}\right] .
\end{aligned}
$$

The transition matrix can be written as follows:

$$
\Phi=\mathbf{D}_{m-1} \mathbf{D}_{m-2} \cdots \mathbf{D}_{1} \mathbf{D}_{0}
$$

According to Floquet theory [31], the chatter stability depends on the eigenvalues of the transition matrix $\Phi$ : if the moduli of all the eigenvalues of the transition matrix $\Phi$ are less than unity, the system is stable; otherwise, it is unstable; that is,

$$
\max (|\Lambda(\boldsymbol{\Phi})|) \begin{cases}<1 & \text { stable } \\ =1 & \text { stable boundary } \\ >1 & \text { unstable. }\end{cases}
$$

When the cutting system is in its critical stable state, its axial cutting depth is called the limiting axial cutting depth. The above formulas show that the limiting axial cutting depth is determined by the normal cutting force coefficient $K_{n}$, the tangential cutting force coefficient $K_{t}$, the modal mass $m$, the modal damping coefficient $c$, the modal stiffness coefficient $k$, the radial immersion ratio $r_{c}$, the spindle speed $\Omega$, and the number of cutting teeth $N$; that is,

$$
\begin{aligned}
& a_{\text {plim }}=\left\{a_{p} \mid \max \left(\left|\Lambda\left(\Phi\left(K_{n}, K_{t}, m, c, k, r_{c}, N, \Omega\right)\right)\right|\right)\right. \\
& \quad=1\} .
\end{aligned}
$$

\section{Reliability Analysis of the Chatter Stability}

Mechanical reliability refers to the ability of mechanical products to complete the required functions under the specified conditions over a specified time period [32-34]. Measuring this ability with probability is reliability. The reliability of the chatter stability refers to the probability that the actual axial cutting depth is less than the limiting axial cutting depth. According to (18), there is no explicit expression for the limiting axial cutting depth. Therefore, the first step is to use a neural network to approximate the functional relationship of the limiting axial cutting depth.

3.1. Establishing a Back-Propagation Neural Network. An artificial neural network usually exhibits a good self-learning ability and a good fault tolerance and can be used for nonlinear mapping. Thus, it is well suited to deal with large amounts of data and to perform complex nonlinear mapping tasks. To date, back-propagation (BP) neural networks are among the most essential and most perfect artificial neural networks.

The design of a BP neural network mainly includes the design of the input layer, the output layer, the hidden layer, and the transformation function.

3.1.1. Design of the Input and the Output Layer. According to the analysis of the chatter stability based on the FDM, the input layer was selected to consist of 9 neurons, which represent the tangential cutting force coefficient $K_{t}$, the normal cutting force coefficient $K_{n}$, the modal damping coefficient $c_{x}$, the modal stiffness coefficient $k_{x}$, and the modal mass $m_{x}$ in the $x$-direction, as well as the modal damping coefficient $c_{y}$, the modal stiffness coefficient $k_{y}$, and the modal mass $m_{y}$ in the $y$-direction, and the radial immersion ratio $r_{c}$, respectively. For the output layer, a singleneuron structure was adopted, which represents the limiting axial cutting depth $a_{\text {plim }}$.

3.1.2. Design of the Hidden Layer. According to HechtNielsen $[35,36]$, a BP neural network with three layers is enough to complete the general mapping. In a three-layer network, the approximate relationship between the number of neurons in the hidden layer $n_{2}$ and the number of neurons in the input layer $n_{1}$ is given by

$$
n_{2}=2 \times n_{1}+1 \text {. }
$$

Therefore, the number of neurons in the hidden layer was selected to be 19 .

3.1.3. Data Normalization. The conversion function of the network is a sigmoid function. Both ends of the output curve of this function are smooth, but in the middle part the curve varies drastically. Therefore, it is necessary to normalize the input and output variables by replacing the original data with corresponding values in the interval $(0,1)$ to achieve a faster convergence rate.

The resulting topological structure of the $\mathrm{BP}$ neural network model used in this study is shown in Figure 2.

The basic algorithm for calculating the milling chatter stability using a BP neural network is as follows. Firstly, $n$ 


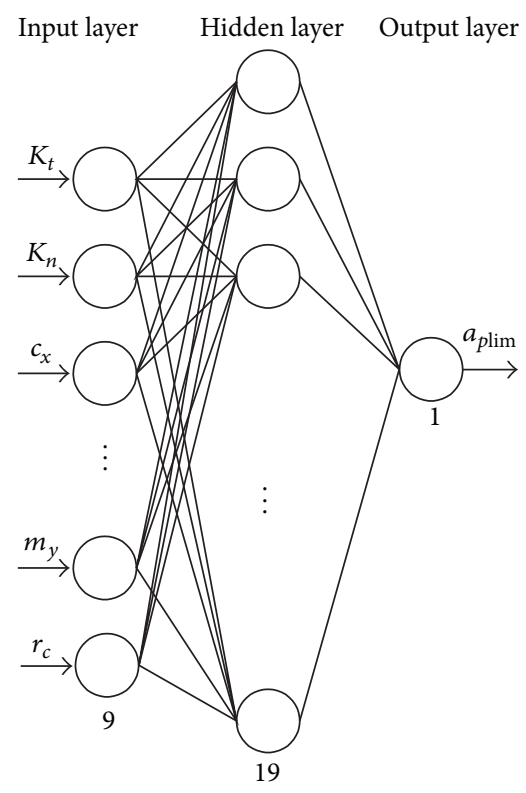

FIgURE 2: Topological structure of the BP neural network model used in this study.

groups of random samples $\mathbf{x}_{i}(i=1,2, \ldots, n)$ are generated from the basic variables (such as the kinetic parameters $\left.K_{n}, K_{t}, c_{x}, c_{y}, k_{x}, k_{y}, m_{x}, m_{y}\right)$. Then, the limiting axial cutting depth $a_{\text {plim }}\left(\mathbf{x}_{i}\right)$ of the random samples can be obtained through numerical analysis. The network grid will be trained using the data $\left\{\mathbf{x}_{i}, a_{\text {plim }}\left(\mathbf{x}_{i}\right)\right\}(i=1,2, \ldots, n)$. The trained network can be used to calculate the limiting axial cutting depth for different values of the basic variables. On the basis of this algorithm, either the Monte Carlo simulation method (MCSM) or the moment method (MM) can be used to determine the reliability of the milling chatter stability. The described algorithm is illustrated in Figure 3.

\subsection{The Monte Carlo Simulation Method. The MCSM offers} a wide universality and is usually fairly easy to implement. Furthermore, as the number of simulations increases, its accuracy will improve.

The basic idea of the MCSM is as follows. Firstly, random samples in the space of the input variables are generated, and then the structural response value is calculated by plugging the random samples into the system model. The probabilistic nature and statistical regulation of the whole system are determined by the probabilistic nature and statistical regulation of the random samples and their responses. The basic steps of the MCSM are as follows:

(1) Based on the distribution characteristics of the basic variables, $n$ groups of random samples $\mathbf{x}_{i}(i=1,2$, $\ldots, n)$ are generated.

(2) The structural response value $g\left(\mathbf{x}_{i}\right)$ is then calculated by plugging $n$ groups of random samples into the reliability analysis model.

(3) The number of samples in the failure domain is counted and denoted as $n_{f}$.

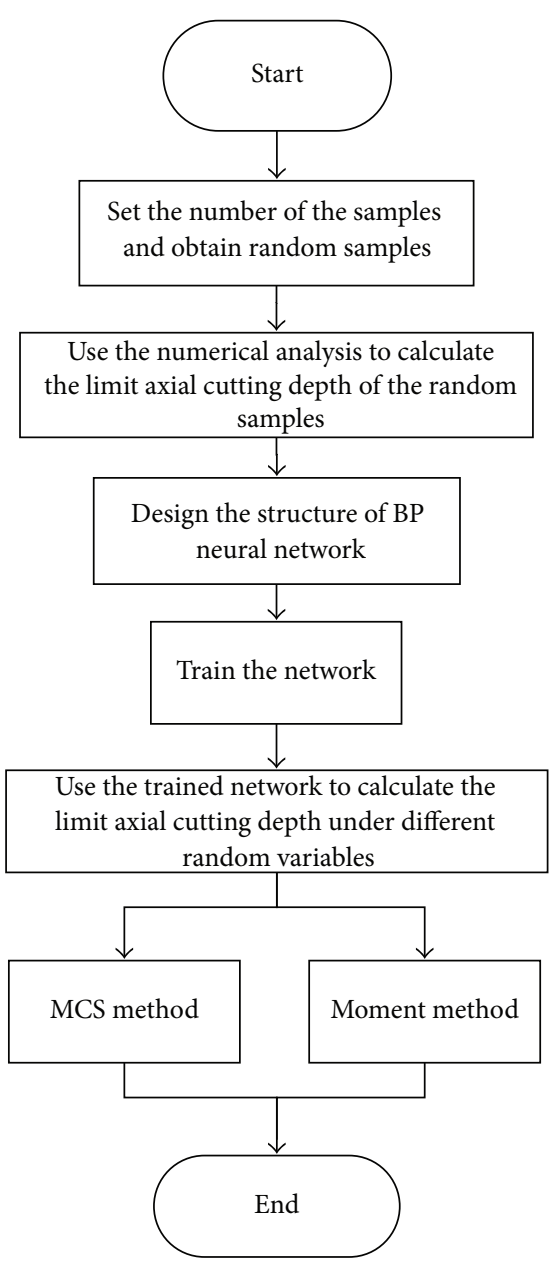

FIGURE 3: Flowchart for calculating the chatter stability reliability utilizing the BP neural network.

(4) The probability of failure is defined as the ratio of the number of samples in the failure region to the total number of samples: that is,

$$
P_{f} \approx \frac{n_{f}}{n} \text {. }
$$

3.3. Moment Method. Although the MCSM is straightforward to implement as it only requires repetitive executions of deterministic simulations, typically a large number of simulations are needed because the solution statistics converge relatively slowly. Therefore, the MCSM is often used as a standard to verify the accuracy of other methods.

In practical engineering, the first-order second-moment method (FOSMM) is the most simple and most commonly used method, but it will generate a large error for higher degree-nonlinear functions. In addition to the gradient of the nonlinear function, the second-order second-moment method (SOSMM) also considers the concavity and curvature of the limit state surface in the vicinity of the design point. Therefore, using the SOSMM can improve the accuracy of the reliability analysis. 
TABLE 1: Dynamic parameters of the simulated milling system and mean values and standard deviations of the corresponding probability distributions.

\begin{tabular}{lccc}
\hline Random variable & Mean value & Standard deviation & Probability distribution type \\
\hline$K_{t}$ & $6 \times 10^{8} \mathrm{~N} / \mathrm{m}^{2}$ & $3 \times 10^{7} \mathrm{~N} / \mathrm{m}^{2}$ & Normal \\
$K_{n}$ & $2 \times 10^{8} \mathrm{~N} / \mathrm{m}^{2}$ & $1 \times 10^{7} \mathrm{~N} / \mathrm{m}^{2}$ & Normal \\
$c_{x}, c_{y}$ & $5.089 \mathrm{~N} \cdot \mathrm{s} / \mathrm{m}$ & $5.089 \times 10^{-2} \mathrm{~N} \cdot \mathrm{s} / \mathrm{m}$ & Normal \\
$k_{x}, k_{y}$ & $1.34 \times 10^{6} \mathrm{~N} / \mathrm{m}$ & $1.34 \times 10^{4} \mathrm{~N} / \mathrm{m}$ & Normal \\
$m_{x}, m_{y}$ & $0.03993 \mathrm{~kg}$ & $0.0003993 \mathrm{~kg}$ & Normal \\
$r_{c}$ & 0.6 & 0.006 & Normal \\
\hline
\end{tabular}

The value of the reliability index $\beta$ corresponds to the distance from the origin to the hyperplane in the standard normal space, which is the geometric meaning of the reliability index $\beta$. Therefore, it is first necessary to normalize the random variable $\mathbf{x}$; that is,

$$
\mathbf{u}=\frac{\mathbf{x}-\mu_{\mathbf{X}}}{\sigma_{\mathbf{X}}} .
$$

The reliability index $\beta$ and the design point $\mathbf{u}^{*}$ can be obtained using the FOSMM. The second-order Taylor expansion of the function $g(\mathbf{u})$ at the design point $\mathbf{u}^{*}$ is

$$
\begin{aligned}
\widetilde{g}(\mathbf{u}) \approx & g\left(\mathbf{u}^{*}\right)+\nabla g\left(\mathbf{u}^{*}\right)^{T}\left(\mathbf{u}-\mathbf{u}^{*}\right) \\
& +\frac{1}{2}\left(\mathbf{u}-\mathbf{u}^{*}\right)^{T} \nabla^{2} g\left(\mathbf{u}^{*}\right)\left(\mathbf{u}-\mathbf{u}^{*}\right),
\end{aligned}
$$

where $\nabla^{2} g\left(\mathbf{u}^{*}\right)$ is the second-order derivative of the function.

The two ends of (22) were then divided by $\left|\nabla g\left(\mathbf{u}^{*}\right)\right|$ :

$$
\tilde{g}(\mathbf{u}) \approx \boldsymbol{\alpha}^{T}\left(\mathbf{u}-\mathbf{u}^{*}\right)+\frac{1}{2}\left(\mathbf{u}-\mathbf{u}^{*}\right)^{T} \mathbf{B}\left(\mathbf{u}-\mathbf{u}^{*}\right),
$$

where

$$
\begin{aligned}
& \boldsymbol{\alpha}=\frac{\nabla g\left(\mathbf{u}^{*}\right)}{\left|\nabla g\left(\mathbf{u}^{*}\right)\right|}, \\
& \mathbf{B}=\frac{\nabla^{2} g\left(\mathbf{u}^{*}\right)}{\left|\nabla g\left(\mathbf{u}^{*}\right)\right|},
\end{aligned}
$$

In order to consider the curvature of the limit state surface, the variables must be converted from the standard normal space $\mathbf{u}$ to the space $\mathbf{y}$. In the space $\mathbf{y}$, the design point $\mathbf{y}^{*}$ falls on the coordinate axis $\left(\mathbf{y}^{*}=\{0,0, \ldots, \beta\}^{T}\right)$. An orthogonal matrix $\mathbf{H}$ can then be constructed so that $\mathbf{y}=\mathbf{H u}$. Now, (23) can be rewritten as

$$
\beta-y_{n}+\frac{1}{2}\left(\begin{array}{c}
y_{n-1} \\
y_{n}-\beta
\end{array}\right) \mathbf{R}\left(\begin{array}{c}
y_{n-1} \\
y_{n}-\beta
\end{array}\right)=0,
$$

where $\mathbf{y}_{n-1}=\left[y_{1}, y_{2}, \ldots, y_{n-1}\right]^{T}$ and $\mathbf{R}=\mathbf{H B H}^{T}$.

Solving the above equation and retaining the first two orders of the solution,

$$
y_{n}=\beta+\frac{1}{2} y_{n}^{T} \mathbf{R}_{n-1} y_{n-1},
$$

where $\mathbf{R}_{n-1}$ is a new matrix consisting of $n-1$ rows and columns of the matrix $\mathbf{R}$. The new approximation of (26) can be obtained by calculating the eigenvalue of the matrix $\mathbf{R}_{n-1}$, denoted as

$$
y_{n}=\beta+\frac{1}{2} \sum_{i=1}^{n-1} w_{i} y_{i}^{2}
$$

where $w_{i}$ is the eigenvalue of the matrix $\mathbf{R}_{n-1}$ and also the curvature of the limit state surface at the design point.

Thus, the failure domain $F$ can be approximated by $\widetilde{F}=$ $\{\widetilde{g}(y)<0\}$. Finally, the probability of failure can be estimated using the method proposed by Breitung [37].

$$
P_{f}=P(\widetilde{F})=\Phi(-\beta) \prod_{i=2}^{n-1}\left(1+w_{i} \beta\right)^{-1 / 2}
$$

\section{Numerical Example}

For this example, we assumed that the dynamic characteristics in the $x$-direction and the $y$-direction of the milling machine system are balanced and symmetrical. The number of the cutter teeth is $N=2$, and the dynamic parameters of the system and mean values and standard deviations of the corresponding probability distributions are listed in Table 1.

The value of the standard deviation of the random parameters should be obtained through the test or the statistical analysis of the experimental data. If the experimental data are not available, the standard deviations of mechanical property parameters could be determined by the variation coefficient [38]. If the random variables are affected by a large number of independent factors, they are generally subject to normal distribution [39].

Substitute mean values of the dynamic parameters $K_{t}$, $K_{n}, c_{x}, c_{y}, k_{x}, k_{y}, m_{x}, m_{y}$, and $r_{c}$ into (17). Then, stability lobes of the milling system can be obtained. To study the effects of variations of variable $X_{i}(i=1,2, \ldots, 9)$ on the stability boundaries, the stability lobes are obtained when $x_{i}=\mu_{X_{i}}-3 \sigma_{X_{i}}$ and $x_{i}=\mu_{X_{i}}+3 \sigma_{X_{i}}$ (other variables are equal to their mean values). All the results are depicted in Figure 4. From the results, the variances of $K_{t}, k_{x}, k_{y}, m_{x}$, and $m_{y}$ have significant influences on the stability boundaries.

The critical axial cutting depth of the system is found to be lowest for a spindle speed $\Omega$ of $10740 \mathrm{r} / \mathrm{min}$; that is, $\min \left(a_{\text {plim }}\right)=9.33 \times 10^{-5} \mathrm{~m}$. At this speed, the possibility of the occurrence of chatter is highest, so we selected this speed to 

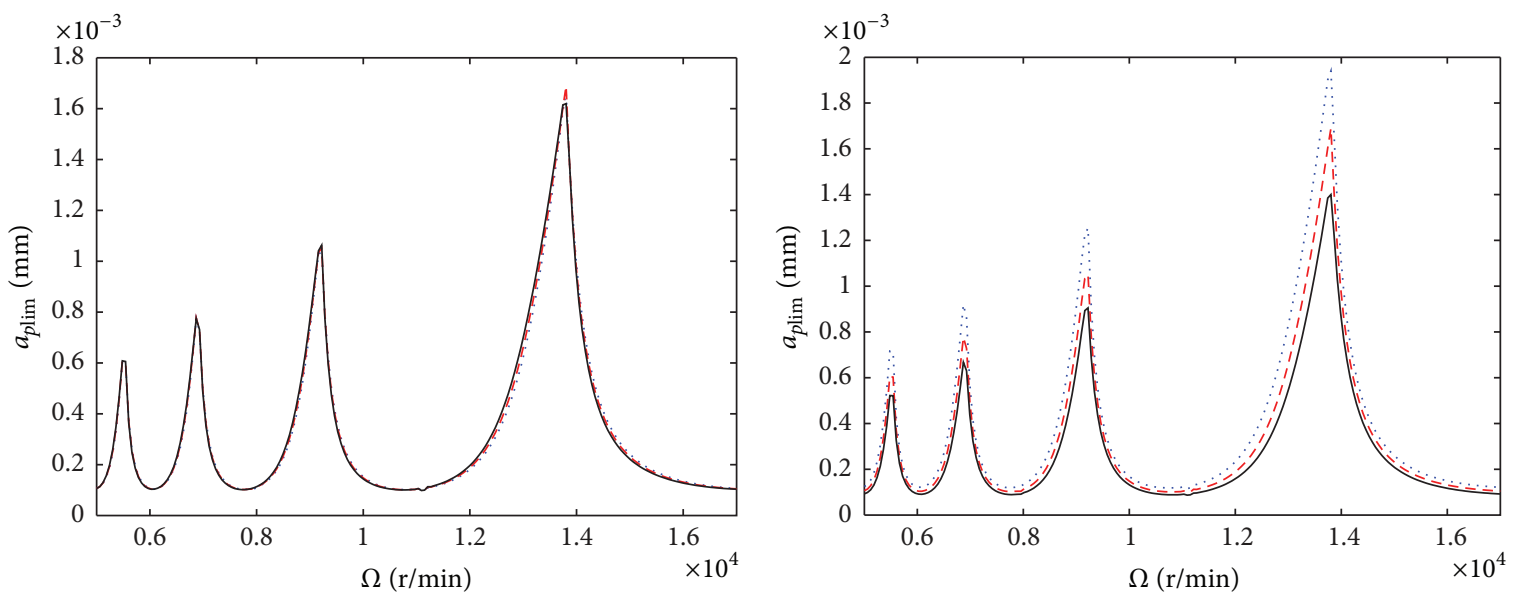

$$
\begin{aligned}
& \ldots . . K_{n}=\mu_{K_{n}}-3 \sigma_{K_{n}} \\
& ---K_{n}=\mu_{K_{n}} \\
& -K_{n}=\mu_{K_{n}}+3 \sigma_{K_{n}}
\end{aligned}
$$$$
\begin{aligned}
\ldots \ldots K_{t} & =\mu_{K_{t}}-3 \sigma_{K_{t}} \\
---K_{t} & =\mu_{K_{t}} \\
-K_{t} & =\mu_{K_{t}}+3 \sigma_{K_{t}}
\end{aligned}
$$

(a)


$$
\begin{aligned}
& \ldots c_{x}=\mu_{c_{x}}-3 \sigma_{c_{x}} \\
& --c_{x}=\mu_{c_{x}} \\
& -c_{x}=\mu_{c_{x}}+3 \sigma_{c_{x}}
\end{aligned}
$$

$$
\begin{aligned}
& \ldots c_{y}=\mu_{c_{y}}-3 \sigma_{c_{y}} \\
& --c_{y}=\mu_{c_{y}} \\
& -c_{y}=\mu_{c_{y}}+3 \sigma_{c_{y}}
\end{aligned}
$$

(c)
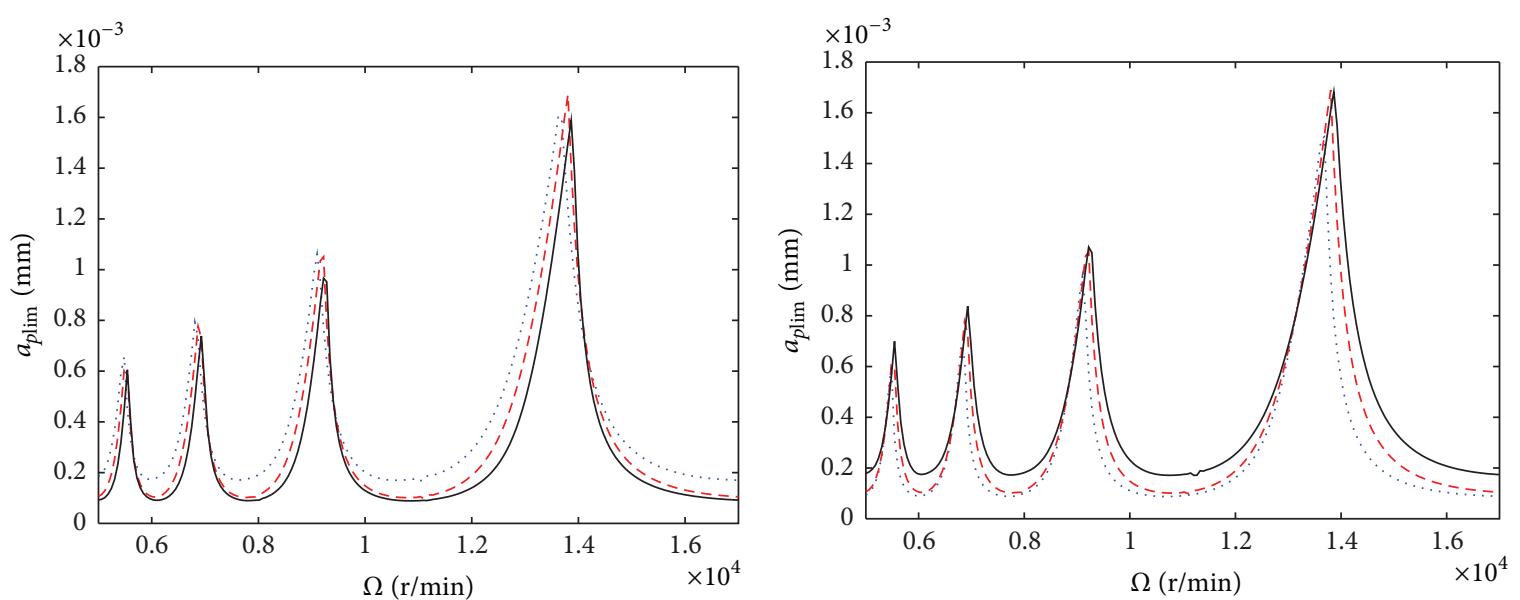

$$
\begin{aligned}
\ldots & k_{x}=\mu_{k_{x}}-3 \sigma_{k_{x}} \\
--k_{x} & =\mu_{k_{x}} \\
-k_{x} & =\mu_{k_{x}}+3 \sigma_{k_{x}}
\end{aligned}
$$$$
\begin{aligned}
\ldots \ldots k_{y} & =\mu_{k_{y}}-3 \sigma_{k_{y}} \\
---k_{y} & =\mu_{k_{y}} \\
-k_{y} & =\mu_{k_{y}}+3 \sigma_{k_{y}}
\end{aligned}
$$

(e)

Figure 4: Continued. 




(g)



(h)



(i)

FiguRE 4: The effects of different variables on the stability boundaries.

TABLE 2: Comparison of the reliabilities obtained using the different methods.

\begin{tabular}{lcccc}
\hline & BP-AFOSM & BP-SOSMM & BP-MCSM & MCSM \\
\hline Reliability & 0.6349 & 0.7070 & 0.6879 & 0.6874 \\
\hline
\end{tabular}

calculate the reliability of the chatter stability. The functional expression of the limiting axial cutting depth is obtained using the BP neural network. The actual axial cutting depth is considered to be equal to the minimum of the critical axial cutting depth of $9.33 \times 10^{-5} \mathrm{~m}$. Then, the MCSM and the MM are used to determine the reliability of the milling chatter stability. The direct MCSM is employed for comparison to demonstrate the validity of the methods based on the BP neural network. The number of direct MCSM samples is 1 $\times 10^{5}$, and it costs about 22 hours ( 0.8 seconds are needed for each deterministic analysis). However, in the proposed method, the number of training samples of the BP neural network is 500, and about 400 seconds is needed for the method based on the BP neural network. After fitting the functional relationship of the limit axial cutting depth, the reliability is computed with the MCSM and MM, respectively. The MCSM needs about 153 seconds, and the AFOSMM needs about 6.4 seconds, and the SOSMM needs about 12 seconds. Therefore, the time consumed by the method based on the BP neural network is significantly less than that of the direct MCSM. The reliabilities obtained using the different methods are compared in Table 2. The accuracy of the methods based on the BP neural network is found to be acceptable. The accuracy of the SOSMM is much higher than that of the advanced first-order second-moment method 
(AFOSMM), but it is still lower than MCSM. In addition, smaller values could be chosen for the actual axial cutting depth to enhance the reliability. For instance, when choosing an actual axial cutting depth of $a_{p}=8 \times 10^{-5} \mathrm{~m}$, the reliability will increase to $R=0.9935$.

\section{Conclusions}

Vibrations are among the main obstacles limiting the accuracy and reliability of milling processes, and chatter is considered the most important vibration in a milling machine. In this paper, the stability of regenerative chatter that might occur during the milling process was analyzed, and a reliability analysis of the chatter stability was performed utilizing a BP neural network. The effect of random factors on the stability of the milling process was analyzed. This approach is more suitable for practical engineering applications than theoretical calculations based on fixed cutting parameters. The reliability of the stability of the milling process was calculated for different spindle speeds using both Monte Carlo simulations and the moment method. The results are considered to be helpful for improving the machining accuracy and machining efficiency of high-speed milling processes.

\section{Competing Interests}

The authors declare that there is no conflict of interests regarding the publication of this paper.

\section{Acknowledgments}

The authors gratefully acknowledge the support of National Natural Science Foundation of China (51575094, 51135003, and 51305071), Program for New Century Excellent Talents in University of Ministry of Education of China (NCET13-0103), and Fundamental Research Funds for the Central Universities (N140304003).

\section{References}

[1] S. Smith and J. Tlusty, "Update on high-speed milling dynamics," Journal of Engineering for Industry, vol. 112, no. 2, pp. 142$149,1990$.

[2] J. Tlusty and F. Ismail, "Basic non-linearity in machining chatter," CIRP Annals_Manufacturing Technology, vol. 30, no. 1, pp. 299-304, 1981.

[3] M. Wiercigroch and A. M. Krivtsov, "Frictional chatter in orthogonal metal cutting," Philosophical Transactions of the Royal Society A: Mathematical, Physical and Engineering Sciences, vol. 359, no. 1781, pp. 713-738, 2001.

[4] M. A. Davies and T. J. Burns, "Thermomechanical oscillations in material flow during high-speed machining," Philosophical Transactions of the Royal Society A: Mathematical, Physical and Engineering Sciences, vol. 359, no. 1781, pp. 821-846, 2001.

[5] Y. Altintas, G. Stépán, D. Merdol, and Z. Dombovari, "Chatter stability of milling in frequency and discrete time domain," CIRP Journal of Manufacturing Science \& Technology, vol. 1, no. 1, pp. 35-44, 2008.
[6] R. Sridhar, R. E. Hohn, and G. W. Long, "A stability algorithm for the general milling process: contribution to machine tool chatter research-7," Journal of Engineering for Industry, vol. 90, no. 2, pp. 330-334, 1968.

[7] S. Smith and J. Tlusty, "Efficient simulation programs for chatter in milling," CIRP Annals-Manufacturing Technology, vol. 42, no. 1, pp. 463-466, 1993.

[8] M. L. Campomanes and Y. Altintas, "An improved time domain simulation for dynamic milling at small radial immersions," Journal of Manufacturing Science \& Engineering, vol. 125, no. 3, pp. 416-422, 2003.

[9] I. Minis and R. Yanushevsky, "A new theoretical approach for the prediction of machine tool chatter in milling," Journal of Engineering for Industry, vol. 115, no. 1, pp. 1-8, 1993.

[10] Y. Altintaş and E. Budak, "Analytical prediction of stability lobes in milling," CIRP Annals-Manufacturing Technology, vol. 44, no. 1, pp. 357-362, 1995.

[11] S. D. Merdol and Y. Altintas, "Multi frequency solution of chatter stability for low immersion milling," Journal of Manufacturing Science \& Engineering, vol. 126, no. 3, pp. 459-466, 2004.

[12] P. V. Bayly, J. E. Halley, B. P. Mann, and M. A. Davies, "Stability of interrupted cutting by temporal finite element analysis," Journal of Manufacturing Science and Engineering, vol. 125, no. 2, pp. 220-225, 2003.

[13] T. Insperger and G. Stépán, "Semi-discretization method for delayed systems," International Journal for Numerical Methods in Engineering, vol. 55, no. 5, pp. 503-518, 2002.

[14] T. Insperger, G. Stépán, and J. Turi, "On the higher-order semidiscretizations for periodic delayed systems," Journal of Sound \& Vibration, vol. 313, no. 1-2, pp. 334-341, 2008.

[15] Y. Ding, L. M. Zhu, X. J. Zhang, and H. Ding, "A full-discretization method for prediction of milling stability," International Journal of Machine Tools \& Manufacture, vol. 50, no. 5, pp. 502509, 2010.

[16] M. Li, G. Zhang, and Y. Huang, "Complete discretization scheme for milling stability prediction," Nonlinear Dynamics, vol. 71, no. 1-2, pp. 187-199, 2013.

[17] Y. Ding, L. M. Zhu, X. J. Zhang, and H. Ding, "Numerical integration method for prediction of milling stability," Journal of Manufacturing Science \& Engineering, vol. 133, no. 3, Article ID 031005, pp. 391-407, 2011.

[18] Y. Ding, L. M. Zhu, X. J. Zhang, and H. Ding, "Stability analysis of milling via the differential quadrature method," Journal of Manufacturing Science and Engineering, vol. 135, no. 4, Article ID 044502, 7 pages, 2013.

[19] F. M. Asl and A. G. Ulsoy, "Analysis of a system of linear delay differential equations," Journal of Dynamic Systems, Measurement, and Control, vol. 125, no. 2, pp. 215-223, 2003.

[20] S. Yi, P. W. Nelson, and A. G. Ulsoy, "Delay differential equations via the matrix Lambert $W$ function and bifurcation analysis: application to machine tool chatter," Mathematical Biosciences and Engineering, vol. 4, no. 2, pp. 355-368, 2007.

[21] E. A. Butcher, O. A. Bobrenkov, E. Bueler, and P. Nindujarla, "Analysis of milling stability by the chebyshev collocation method: algorithm and optimal stable immersion levels," Journal of Computational and Nonlinear Dynamics, vol. 4, no. 3, pp. 1-12, 2009.

[22] E. A. Butcher, H. Ma, E. Bueler, V. Averina, and Z. Szabo, "Stability of linear time-periodic delay-differential equations via Chebyshev polynomials," International Journal for Numerical Methods in Engineering, vol. 59, no. 7, pp. 895-922, 2004. 
[23] P. Albertelli, N. Cau, G. Bianchi, and M. Monno, "The effects of dynamic interaction between machine tool subsystems on cutting process stability," The International Journal of Advanced Manufacturing Technology, vol. 58, no. 9-12, pp. 923-932, 2012.

[24] A. Iglesias, J. Munoa, and J. Ciurana, "Optimisation of face milling operations with structural chatter using a stability model based process planning methodology," International Journal of Advanced Manufacturing Technology, vol. 70, no. 14, pp. 559-571, 2014.

[25] M. H. Kurdi, T. L. Schmitz, R. T. Haftka, and B. P. Mann, "A numerical study of uncertainty in stability and surface location error in high-speed milling," ASME International Mechanical Engineering Congress \& Exposition, vol. 16, no. 1, pp. 387-395, 2005.

[26] G. S. Duncan, M. H. Kurdi, T. L. Schmitz, and J. P. Snyder, "Uncertainty propagation for selected analytical milling stability limit analyses," Transactions of the North American Manufacturing Research Institute of SME, vol. 34, pp. 17-24, 2006.

[27] E. Graham, M. Mehrpouya, and S. S. Park, "Robust prediction of chatter stability in milling based on the analytical chatter stability," Journal of Manufacturing Processes, vol. 15, no. 4, pp. 508-517, 2013.

[28] Y. Liu, L. L. Meng, K. Liu, and Y. M. Zhang, "Chatter reliability of milling system based on first-order second-moment method," International Journal of Advanced Manufacturing Technology, 2016.

[29] W. S. Lin, "The reliability analysis of cutting tools in the HSM processes," Archives of Materials Science \& Engineering, vol. 30, no. 2, pp. 97-100, 2008.

[30] J. Gradišek, M. Kalveram, T. Insperger et al., "On stability prediction for milling," International Journal of Machine Tools and Manufacture, vol. 45, no. 7-8, pp. 769-781, 2005.

[31] M. Farkas, Periodic Motions, vol. 104 of Applied Mathematical Sciences, Springer, New York, NY, USA, 1994.

[32] L. Changyou, W. Wei, Z. Yimin, G. Song, L. Zhenyuan, and Q. Changshuai, "Indexing accuracy reliability sensitivity analysis of power tool turret," Maintenance and Reliability, vol. 17, no. 1, pp. 27-34, 2014.

[33] Y. Chu, Z. Yuan, and J. Chen, "Research on dynamic reliability of a jet pipe servo valve based on generalized stochastic Petri nets," International Journal of Aerospace Engineering, vol. 2015, Article ID 171642, 8 pages, 2015.

[34] Y. Pang, H.-Z. Huang, N.-C. Xiao, Y. Liu, and Y.-F. Li, "Possibilistic reliability analysis of repairable system with omitted or delayed failure effects," Maintenance and Reliability, vol. 14, no. 3, pp. 195-202, 2012.

[35] R. Hecht-Nielsen, "Theory of the backpropagation neural network," Neural Networks, vol. 1, no. 1, pp. 65-93, 1988.

[36] R. Hecht-Nielsen, "Kolmogorov's mapping neural network existence theorem," in Proceedings of the 1st IEEE International Conference on Neural Network, pp. 11-14, San Diego, Calif, USA, 1987.

[37] K. Breitung, "Asymptotic approximations for multinormal integrals," Journal of Engineering Mechanics, vol. 110, no. 3, pp. 357366, 1984.

[38] Y. Zhang, X. He, Q. Liu, and B. Wen, "Robust reliability design of Banjo flange with arbitrary distribution parameters," Journal of Pressure Vessel Technology, vol. 127, no. 4, pp. 408-413, 2005.

[39] P. D. T. O'Connor and A. Kleyner, Practical Reliability Engineering, John Wiley \& Sons, Chichester, UK, 5th edition, 2012. 


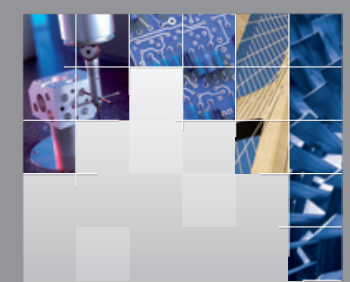

\section{Enfincering}
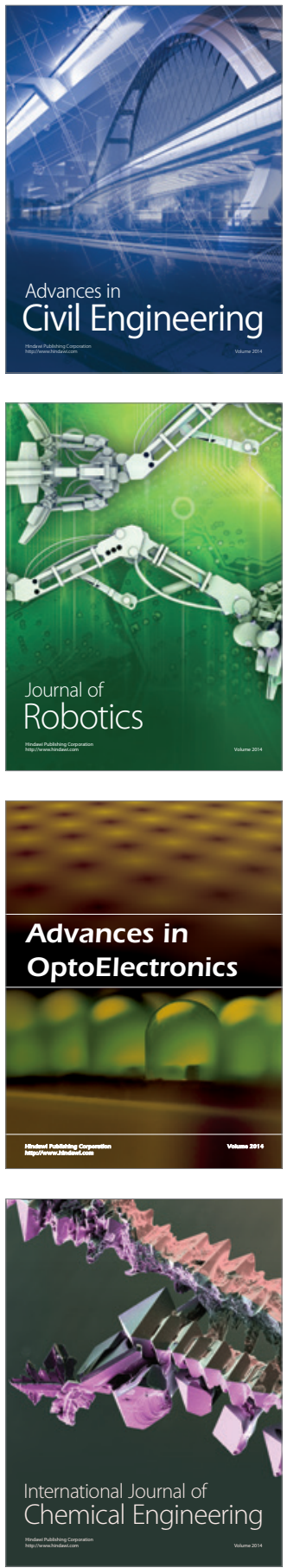

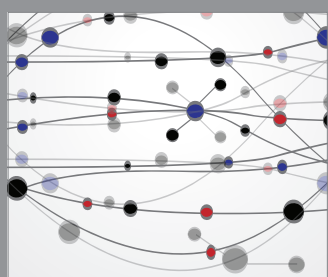

The Scientific World Journal

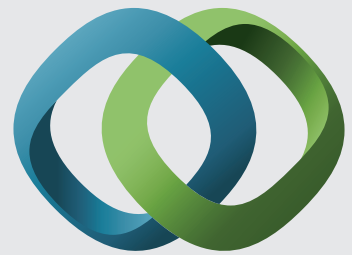

\section{Hindawi}

Submit your manuscripts at

http://www.hindawi.com
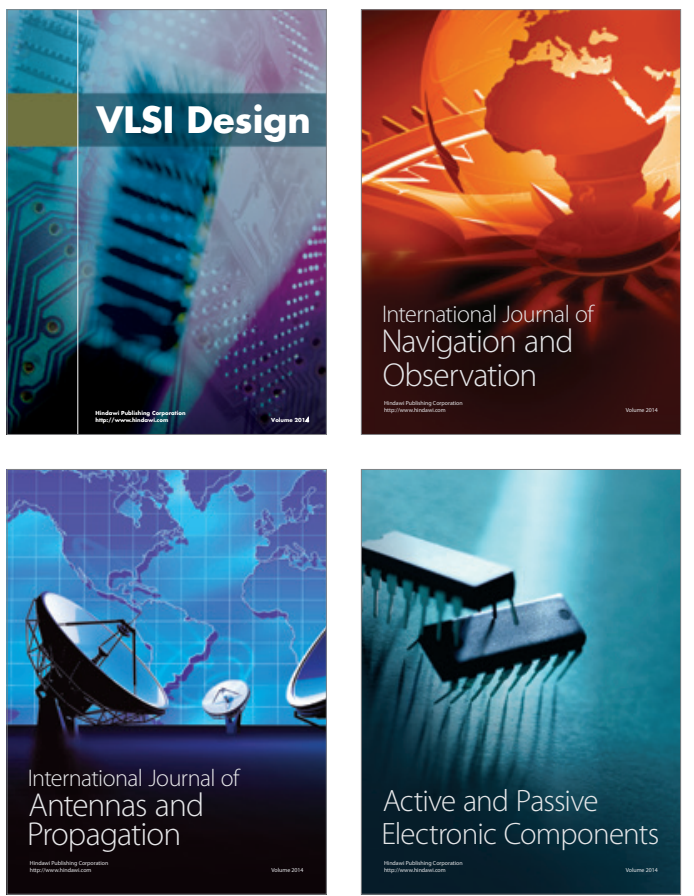
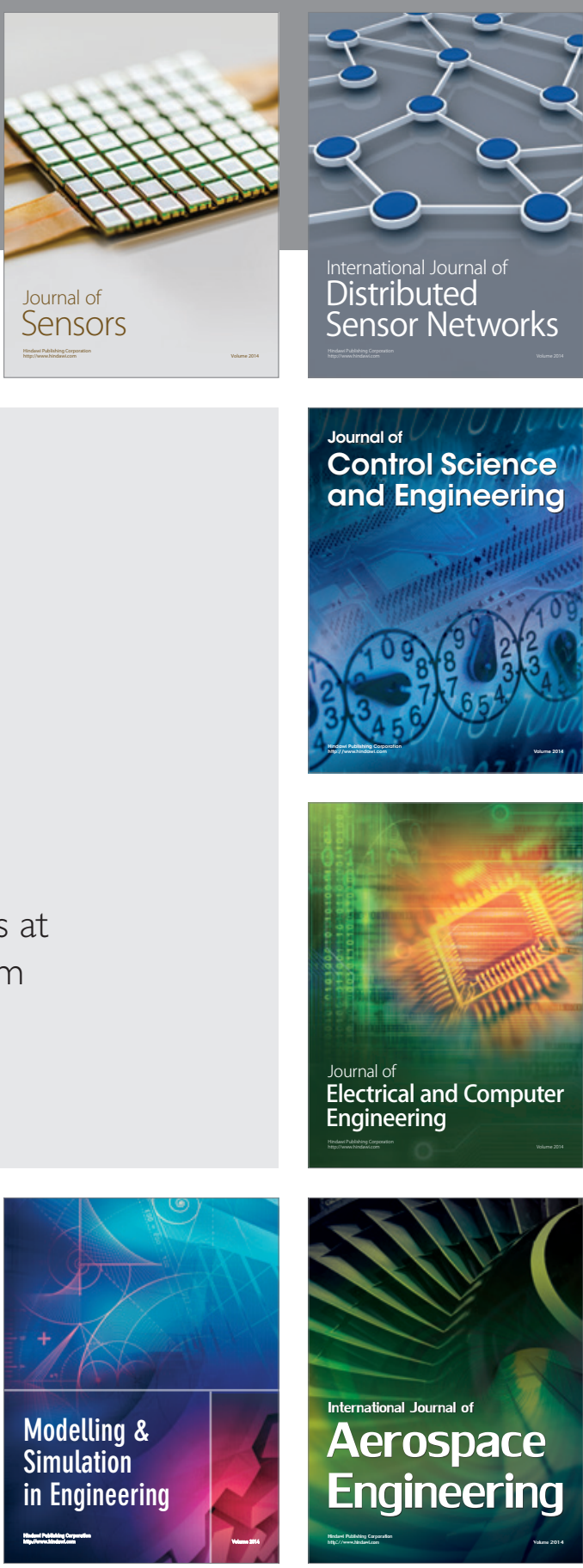

International Journal of

Distributed

Sensor Networks

Journal of

Control Science

and Engineering
